\title{
Never straightforward
}

\author{
Stephen Hancocks OBE \\ Editor-in-Chief
}

The BDJ Upfront section includes editorials, letters, news, book reviews and interviews. Please direct your correspondence to the News Editor,

Kate Quinlan at k.quinlan@nature.com. Press releases or articles may be edited, and should include a colour photograph if possible.

$\mathrm{T}$ he pandemic has given us all the chance to reflect. This may be as a direct effect of being grounded at home by lockdowns, isolation, self-isolation, shielding or confinement by dint of other hitherto unknown terminology. It may be due to 'just doing less' for much the same reasons or it may be prompted by the substantially altered patten of our working lives. I had previously opined on more than one occasion that dentistry in the coming years would be gradually but then substantially different to the traditional landscape. I of course had no inkling, however, of the radical twist that the advent of coronavirus was to deliver or the opportunity it would provide to give us a circuit break for contemplation.

We are having our sensitised fingertips cruelly pressed down on an uncertain braille; attempting to map the unknown onto a previous, if precarious, predictability. What will remain the same is the way in which dentistry and our delivery of it will be a part of the weave of society and be affected by it. Broadly within this context, the future will fall into the immediate post-pandemic space and then a medium-term consolidation, which will be a mix of corona-moderated hangovers and a return to something like the pattern being laid out prior to 2020 .

In predicting a different future for dentistry, I had hitherto based much of my analysis on falling caries rates, and for the most part, I suspect that the underlying trend will continue. However, the large and growing backlog of routine care is inevitably going to create an aberrant bow wave of activity - as indeed will be similarly depicted by so many graphs, histograms and bar charts showing wayward deviations for the 2019-2022 period; everything from redundancies to puppy sales. There is no doubt that pent-up demand and untreated caries left to fester throughout the pandemic will create a blip in restorative provision, from the smallest occlusal preparations through to extractions and replacements, running the gamut from implants to dentures and everything in between. This too, in due course, will accumulate fascinating data and fathomless runes for epidemiologists and others to ponder over for decades to come. Indeed, one wonders, invoking the memory of sadly missed Jimmy Steele, whether this may not lead to a mini revisit to the heavy metal generation; perhaps an unanticipated farewell tour?

The hope is that the backlog might be managed with a twenty-first-century rationale of minimum intervention dentistry (MID), much promoted by this journal, rather than a hasty reversion to a twentieth-century heritage. At a time when a popular clamour an important aspect of practice and, as papers in this issue illustrate, pain control remains an important driver for definitive care as well as patient satisfaction - the opium crisis in US dentistry being a fallout of this and perhaps ironically mirroring the historical infamy of a flamboyant American practitioner, who legally changed his first name from Edgar to become 'Painless' Parker in order to avoid advertising restrictions.

Thankfully, we have been able to grow away from focusing solely on this physiological necessity to providing comprehensive care, with other considerations moving in to take up the void. In planning and managing our futures, we now have to be cognisant of a huge raft of

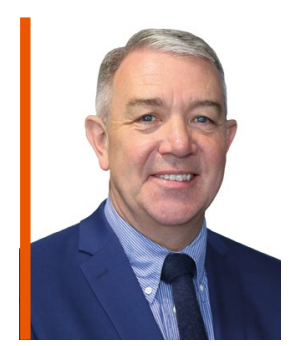

\section{'There is an even greater imperative to pay deeper attention to that which has been, in order to learn and change that which can be.'}

would have us rewrite many episodes in history, there is an even greater imperative to pay deeper attention to that which has been, in order to learn and change that which can be. In this instance the opportunity will exist both in the course of dealing with the disease bulge and in its aftermath to apply the preventive aspects of MID as a contrast to that which has gone before. This can only be to the benefit of patients, oral health, general health and us all.

This needs to be set against what continues to fascinate me, which is the ever-compounding complexity of providing dental care in the modern world. In the setting of the medieval town square, the guiding marketing adjective was 'painless'. The absence of pain was the most important element of the available service. This is still regulation and operating procedures as well as, quite rightly, social awareness of race, gender and equality; balancing our own health and wellbeing and that of our team members against work-related stress.

The hiatus that we have been given by the circumstance of the pandemic to reflect on all of this, and to begin to plot a renewed approach to what we do with a greater regard to value and values, is a once-in-a-lifetime opportunity. It is frightening, it is uncertain and it is not straightforward - the lesson of life is that what seems to be straightforward rarely turns out to be - but if we are wise, we will use it to help us envision a better time to come and one which better represents a world we would prefer to inhabit.

https://doi.org/ 10.1038/s41415-021-2670-3 